\title{
Differences in Pes Planus and Pes Cavus subtalar eversion/inversion before and after prolonged running, using a two-dimensional digital analysis
}

\author{
Charlotte Sinclair', Ulla Svantesson ${ }^{2,3}$, Rita Sjöström4 , Marie Alricsson ${ }^{1,3, *}$ \\ 'Department of Sports Science, Linnaeus University, Kalmar, Sweden \\ ${ }^{2}$ Sahlgrenska Academy, Institute of Neuroscience \& Physiology, University of Gothenburg, Gothenburg, Sweden \\ ${ }^{3}$ Swedish Winter Sport Research Centre, Department of Health Sciences, Mid Sweden University, Östersund, Sweden \\ ${ }^{4}$ Region Jämtland Härjedalen, Unit of Research Education \& Development, Östersund, Sweden
}

In sports, there is a constant discussion about the hyper-pronation and supination of the foot during loading and its relation to injuries or discomfort. The purpose of the present study was to investigate the possible differences in the subtalar joint in the midstance phase of running, between individuals with Pes Planus and Pes Cavus, after 5 min and 45 min of running. Thirty-four subjects, meeting the requirements for Pes Planus ( 30 feet) and Pes Cavus ( 35 feet), according to the criteria for Medial Longitudinal Arch-angle, were included in the study. The calcaneal vertical angle, representing the eversion/inversion of the subtalar joint, was measured using with two-dimensional digital analysis and Dartfish Software with the subjects running barefoot on a treadmill, before and after 45 min of outside running wearing shoes. Both individuals with Pes Cavus and Pes Planus showed a significant increase in the calcaneal eversion $(P<0.05)$ after 45 min of running. Between the groups, there was a significantly greater eversion of the Pes Planus, on the right foot, after 45 min of running $(P<0.05)$ compared to the Pes Cavus. The effect of fatigue evident in the present study suggests that further biomechanical research should be considered when exposing the foot to the repetitive nature of running, conditions most likely responsible for the overrepresented overuse injuries among runners.

Keywords: Calcaneus deviation, Fatigue, Foot, Pronation, Subtalar joint, Supination

\section{INTRODUCTION}

In sports, there are constant discussions about hyperpronation and supination of the foot during load and its relation to injuries or discomfort. The terms refer to an excessive compensatory pronation and supination that are not within the scope of normal absorption and/or surface adaptation or the prolonged phases of the movements (Kannus, 1992).

In clinical practice, it is common to use the foot structure as a predictor of its behavior under load. A previous study reported that the structure of the Medial Longitudinal Arch (MLA) and the position of the subtalar joint (STJ) axes affect each other independently, were the MLA is lowered through the STJ eversion and vice versa (Subotnick, 1974). Based on this theory, Pes Planus (PP) is often described as being more mobile and developing into hyperpronation, while Pes Cavus (PC) is more rigid and develops into hypersupination. Pes Rectus rarely develops excessive compensatory movement patterns. However, neither a characteristic mobility nor a characterized biomechanical compensation pattern under load (referring to the excessive or nonexcessive supination or pronation) of a PC and PP has previously been established. Lack of consistency in previous research and difficulties in comparing the results of the studies due to the use of various measurement methods have been suggested as reasons (Williams and McClay, 2000).

There is no overall used measurement method to examine the foot structure and behavior. In the clinic, it is most common to
*Corresponding author: Marie Alricsson (D) http://orcid.org/0000-0001-6653-3414 Department of Sports Science, Linnaeus University, SE-391 82 Kalmar, Sweden Tel: +46-73-8012299, Fax +46-63-165626, E-mail: marie.alricsson@lnu.se Received: January 10, 2017 / Accepted: April 2, 2017
This is an Open Access article distributed under the terms of the Creative Commons Attribution Non-Commercial License (http://creativecommons.org/licenses/by-nc/4.0/) which permits unrestricted non-commercial use, distribution, and reproduction in any medium, provided the original work is properly cited. 
visually observe the foot; however, since these observations have shown low reliability even among experienced investigators (Razeghi and Batt, 2002), authors in earlier studies recommend standardized measurements (Razeghi and Batt, 2002; Williams and McClay, 2000).

One common foot structure measurement is the MLA-angle, based on three points: medial malleolus, tuberositas ossis navicularis and caput metatarsale 1 (Dahle et al., 1991; Nilsson et al., 2012; Razeghi and Batt, 2002).

The Measurements of dynamic foot behavior during the stance phase of running are often investigated by two-dimensional (2D) digital analysis. The frontal plane motion in the STJ has been described as particularly important during loading, especially in the midstance phase of running where it is a main component of pronation and supination. The calcaneal vertical angle (calcaneus to the ground) (CV-angle) (Hunt et al., 2000; Johanson et al., 1994) is one of many clinical measurements of the STJ frontal plane motions, where eversion/inversion are referred to as a calcaneal deviation in a valgus/varus direction (Johanson et al., 1994).

Both the CV-angle under the stance phase of running (Borel et al., 2011; Johanson et al., 1994; Stacpoole-Shea et al., 1998) and the MLA-angle during walking (McPoil and Cornwall, 2005) have been measured on a $2 \mathrm{D}$ digital freeze-frame image with a digital angle measurement program. The $\mathrm{CV}$-angle has shown excellent intrarater reliability (intraclass correlation $[$ ICC $]=1.00$ ) (Johanson et al., 1994) under the midstance phase of running. The MLA-angle has shown excellent intrarater reliability (ICC $=0.96$ and 0.95 ) and substantial interrater reliability during walking (ICC $=0.67$ ), with standard error of measurement of 0.8 grades, interpreted as extremely low (McPoil and Cornwall, 2005).

In their study, Kaufman et al. (1999) presented an increased risk of injuries in high- and low-arched runners compared to those with normal arches. Meanwhile sports medicine literature has blamed excessive pronation as the cause for nearly all maladies of the lower extremities, further supported in other studies (Dahle et al., 1991; Kannus, 1992). Finding typical foot structures validated to the dynamic behaviour during running could provide the clinician with a valuable and quick assessment tool to separate abnormal structures (referring to PC and PP) and predispose abnormal biomechanics (referring to hyperpronation and hypersupination) in order to plan a suitable treatment. However, there is no evidence that a specific foot structure or dynamic foot behavior during loading means an increased risk of injury (Buldt et al., 2013). Without a consensus on what factors predisposes to injuries, a validation tool for specific types of foot structure and dy- namic behavior would obviously lack clinical meaning.

Overuse injuries are the main reasons for running related injuries and are obviously developed from the repetitive stride cycles, characterized in running (Abt et al., 2011). Some researchers have questioned the validation between a short running analysis and the actual repetitive nature of running, as lasting for several minutes or hours. For example, Dierks et al. (2011) revealed that subjects with patellofemoral pain syndrome did not show any compensation patterns such as knee valgus until they reached a state of fatigue. This is a great indication of the need for further studies investigating runners in a fatigued state. The influence on a midstance STJ motion by running has been investigated in several studies, showing both significant (Clansey et al., 2012; Derrick et al., 2002; Dierks et al., 2010) and nonsignificant alterations (Abt et al., 2011; Hamill et al., 1988).

However, most studies use exhaustive running protocols. Exploring the foot under conditions representing the individual's regular running session might reveal new important information about abnormal foot structure, and abnormal biomechanics and what factors predispose one to injuries.

Therefore, the aim of the present study was to investigate the possible differences in the frontal plane movement (eversion/inversion) in the STJ among individuals with PP and PC, before and after a 45 -min run.

The first hypothesis was that both individuals with PC and PP would be affected by the 45-min running session, showing an increased eversion at the midstance phase. Furthermore, the second hypothesis was that the eversion of PP would be more increased after the running session, compared to PC.

\section{MATERIALS AND METHODS}

\section{Participants}

The participants volunteered on a public local running website. Thirty-four healthy participants, with one or both feet measured as PP (35 feet) or PC (30 feet), according to the criteria for MLA-angle, (PP, $90^{\circ}-130^{\circ}$; PC, $150^{\circ}-180^{\circ}$ ), were included in the study (Dahle et al., 1991). Women and men between the ages of 18 to 65 were considered for participation. The participants had to be familiar with running on a treadmill. Musculoskeletal disorders and pathological disorders affecting movement and function of the lower limbs or a history of pain or injury in the lower limbs during the test period and the past 3 months prior were considered as exclusion criteria.

Ethical approval was obtained from the Ethical Advisory Board 
in South East Sweden (Dnr EPK 204-2014), and the study was conducted in accordance with the Declaration of Helsinki for Human studies.

\section{Instruments}

In the present study, a digital video camera (Andersson CAM 1.5, Net on net, Göteborg, Sweden) with $50 \mathrm{~Hz}$ and a shutter speed of 1/2,000 sec was used in combination with the Dartfish software (Dartfish Live v.5.5, Dartfish, Fribourg, Switzerland). The camera was positioned at a height of 2 inches from the test surface and three feet behind and perpendicular to the object (StacpooleShea et al., 1998). Dartfish is a computer-based software program with video technology to facilitate movement analysis. It enables the examiner to use slow-motion technology to take freeze frame images and take measurements such as angles, distances and timing directly on the still images (Borel et al., 2011).

\section{Procedures}

Only one researcher, with 6 years of clinical experience with foot examinations, was involved in the test procedure. However, the researcher was blinded to group affiliation and order of test occasion, with help from an external person. The test procedure involving the recordings was done on one day, but the measurements on Dartfish were made the following day.

\section{Measurement of MLA-angle}

No study investigating the reliability of Dartfish software measuring the CV- or MLA-angle has been found. A test-retest was done on the MLA-angle measurement, in 10 individuals in order to classify the group of affiliation, PP or PC, and the percentage agreement was measured to be $90 \%$.

The participants stood on one leg, loading the body weight and keeping a hand on a railing for stability. The foot was placed behind previously marked front and side-lines on the treadmill; while in this position, the investigator palpated and marked the reference points on the participant's skin with a fine ballpoint pen (Razeghi and Batt, 2002). The exact points on the metatarsal head, the navicularis and the malleolus were adapted according to Nilsson et al. (2012). The still images were taken of the foot's medial side in the sagittal plane directly after the reference setting. The digital goniometry in Dartfish allowed the examiner to manually work with the computer mouse, in order to insert lines representing the goniometric arms. The end- and start-point of the lines were seen as a cross on the screen, and the lines were drawn between the reference points to form a MLA-angle (Fig. 1).

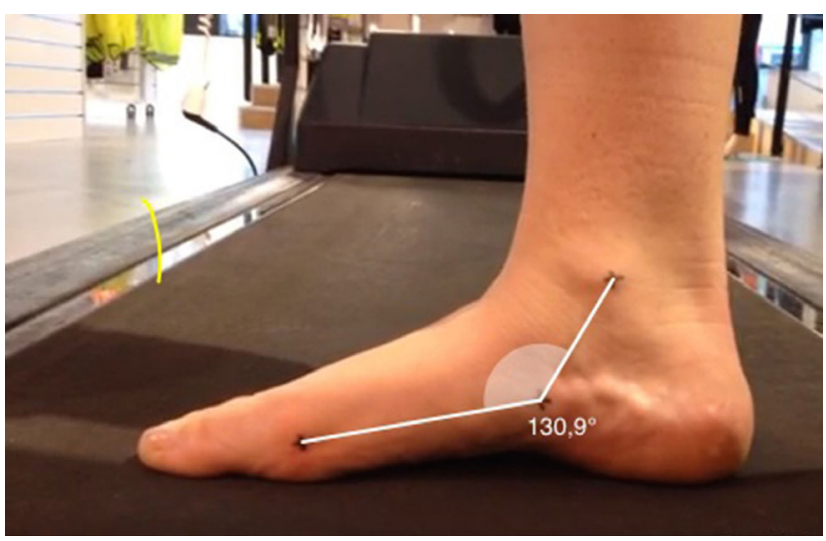

Fig. 1. Still image of the foot's medial side in the sagittal plane, with the Medial Longitudinal Arch-angle as a result of the degrees of the screen.

\section{Measurement of CV-angle}

A pilot study was performed with a test-retest reliability protocol with 10 individuals, on the $\mathrm{CV}$-angle measured during the midstance on two different occasions. The procedure was exactly the same as in the present study, including marking the reference points on the foot and the Dartfish measurement. The interrater reliability, measured with Spearman correlation coefficient, was excellent (left foot, $r_{s}=0.92$; right foot, $r_{s}=0.79$ ). The intrarater reliability was excellent for the right foot $\left(r_{s}=0.96\right)$ and moderate for the left foot $\left(r_{s}=0.75\right)$. While the participant was lying in the prone position, the midline of the calcaneus was marked with a fine ballpoint pen, all according to Elveru et al. (1988).

The participants ran barefoot on the treadmill at a speed of 12 $\mathrm{km} / \mathrm{hr}$ and had been asked to try barefoot running on a treadmill twice during the 2 weeks prior to the test. The recording started after allowing the participant $4 \mathrm{~min}$ to get used to the treadmill running, 1 min of rest and another 1 min of running, and lasted approximately $10 \mathrm{sec}$. Within $10 \mathrm{~min}$ after the run, the participant put on regular running shoes and started to run for $45 \mathrm{~min}$ on plain ground and asphalt at a comfortable jogging pace. After 45 min of running, the participants marked the exertion level by rating of perceived exertion (RPE) according to the Borg scale (Borg, 1998). Within $5 \mathrm{~min}$ after the running session ended, the same barefoot treadmill running procedure was performed at $12 \mathrm{~km} / \mathrm{hr}$.

The slow motion function and the integrated stopwatch in Dartfish allowed the examiner to time the stance phase (heelstrike to toe off) and detect the exact moment that it was $50 \%$ of the stance-phase, which was caught on a freeze frame image, representing the foot and the lower leg in a posterior view. The program's grid was used in order to create a square, around which the lower horizontal line was placed parallel and level to the ground. 
The function to manually insert the lines representing the goniometric arms over the horizontal base in the square and the other one over the line and in the same slope as the previously marked center line on the calcaneus, all the way to the square outline (Fig. 2). The results of the calcaneal vertical angle were divided into the following manners of operation: $90^{\circ}$ angle to the horizontal base (zero position, no movement), over $90^{\circ}$ (varus, inversion) and less than $90^{\circ}$ (valgus, eversion).

\section{Statistical analysis}

Analytical, descriptive, statistics and 95\% confidence interval were used in the study. The analyses were performed in IBM SPSS Statistics ver. 22.0 (IBM Co., Armonk, NY, USA). Since no normal distribution could be predicted, and due to the small group size, nonparametric tests were performed. To test the differences within each group, Wilcoxons signed ranks test was used, and to

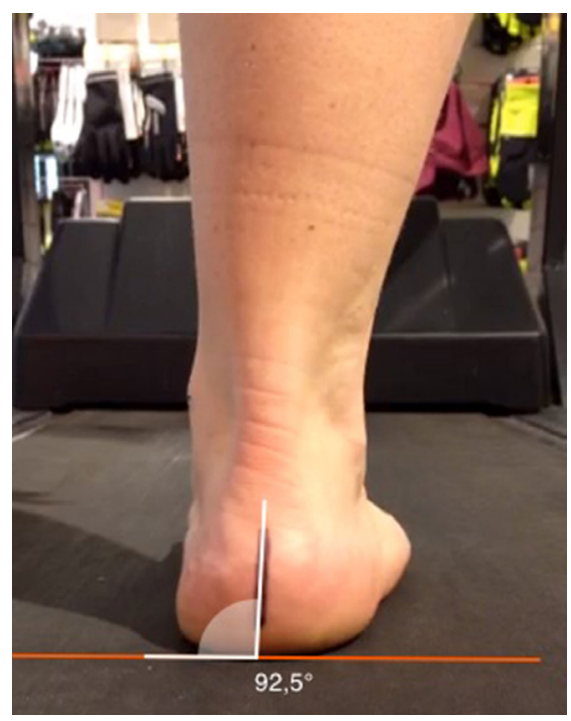

Fig. 2. Still image of calcaneus in the posterior view and the angle between the base and the calcaneus, calcaneal vertical angle. test between the group differences, Mann-Whitneys $U$-test was used. Correlations between the measurement and the individual parameters were calculated with Spearman correlation coefficient. The level of significance was set at $5 \%$.

\section{RESULTS}

Descriptive data on all of the participants in the study group are presented in Table 1.

The results of the $\mathrm{CV}$-angle, measured after $5 \mathrm{~min}$ and $45 \mathrm{~min}$ of running, and the differences between these two protocols are shown in Table 2A. Values lower than $90^{\circ}$ represent eversion and greater values represent inversion. Table $2 \mathrm{~B}$ shows the values for the PP experimental group and Table $2 \mathrm{C}$ presents the measurements for the PC group.

There were no significant differences between the two groups $\mathrm{PC}$ and PP, after 5 min of running, suggesting that there was no relationship between the foot structure and the STJ frontal plane motion. The PP group showed significantly greater eversion on the right foot, after $45 \mathrm{~min}(P<0.05)$ compared to the PC group. No significant differences were found between five to $45 \mathrm{~min}$ of running, in either group, suggesting that the results of our study show a tendency that individuals with low arches have a greater eversion after 45 min of running but not after the 5-min run.

Both the PC group and the PP group showed significantly increased eversion on both their feet $(P<0.05)$ after 45 min of running. This suggests that our hypothesis that the foot is affected by a running session, showing increased eversion in spite of high or low arches, is true.

There was a positive significant correlation $(r=0.46, P<0.006)$ between the individual's own experience of running habits and the measurement of the $\mathrm{CV}$-angle of the right foot after $45 \mathrm{~min}$ of running. Thus, individuals with less running habits showed a greater amount of eversion after 45 min of running than those with more

Table 1. Description of the study population

\begin{tabular}{lcc}
\hline Variable & Study group $(\mathrm{n}=34)$ & Male $(\mathrm{n}=14)$ \\
\hline Age $(\mathrm{yr})$, mean (range) & $36.7(22-55)$ & $42.6(25-55)$ \\
Height (cm), mean (range) & $173(144-198)$ & $182(170-198)$ \\
Weight (kg), mean (range) & $67(44-86)$ & $77(65-86)$ \\
BMl (kg/m²), mean (range) & $22.6(18.7-26)$ & $23.4(21.1-25.6)$ \\
Foot size (cm), median (range) & $40(35-45)$ & $43(40-45)$ \\
Habit of running*, median (range) & $4(1-5)$ & $3(1-5)$ \\
Borgsscale ${ }^{\dagger}$, median (range) & $13(10-18)$ & $13(10-18)$ \\
\hline
\end{tabular}

BMl, body mass index.

*Number of running sessions per week: 1, 0; 2, 1 time/wk; 3, 2-3 times/wk; 4, 4-5 times/wk; 5, or more. 'Level of effort 6-20. 
Sinclair C, et al. • Subtalar eversion/inversion before and after prolonged running

Table 2A. The angle in degrees between the calcaneus and ground of all subjects after 1 min and 45 min of running

\begin{tabular}{|c|c|c|c|c|c|}
\hline \multicolumn{3}{|c|}{ Left foot $(n=34)$} & \multicolumn{3}{|c|}{ Right foot ( $n=31)$} \\
\hline $1 \mathrm{Min}$ & $45 \mathrm{Min}$ & Diff & $1 \mathrm{Min}$ & $45 \mathrm{Min}$ & Diff \\
\hline $87 \pm 2.40$ & $85 \pm 2.90$ & $-2 \pm 1.70$ & $86.7 \pm 3.33$ & $85.1 \pm 4.00$ & $-1.8 \pm 2.03$ \\
\hline
\end{tabular}

Table 2B. The angle in degrees between the calcaneus and to the ground in the experimental group Pes Planus after 1 min and 45 min of running

\begin{tabular}{|c|c|c|c|c|c|}
\hline \multicolumn{3}{|c|}{ Left foot $(n=15)$} & \multicolumn{3}{|c|}{ Right foot $(n=15)$} \\
\hline $1 \mathrm{Min}$ & $45 \mathrm{Min}$ & Diff & $1 \mathrm{Min}$ & $45 \mathrm{Min}$ & Diff \\
\hline $86.7 \pm 2.42$ & $84.6 \pm 2.52$ & $-2.1 \pm 1.69$ & $85.5 \pm 2.78$ & $83.6 \pm 3.65$ & $-1.9 \pm 2.42$ \\
\hline
\end{tabular}

Table 2C. The angle in degrees between the calcaneus and to the ground for the experimental group Pes Cavus after 1 min and 45 min of running

\begin{tabular}{|c|c|c|c|c|c|}
\hline \multicolumn{3}{|c|}{ Left foot $(n=19)$} & \multicolumn{3}{|c|}{ Right foot $(n=16)$} \\
\hline $1 \mathrm{Min}$ & $45 \mathrm{Min}$ & Diff & $1 \mathrm{Min}$ & $45 \mathrm{Min}$ & Diff \\
\hline $87.3 \pm 2.46$ & $85.4 \pm 3.29$ & $-1.9 \pm 1.78$ & $87.7 \pm 3.47$ & $86.3 \pm 3.95$ & $-1.7 \pm 1.74$ \\
\hline
\end{tabular}

Values are presented as mean \pm standard deviation. Diff, difference.

running habits. A positive significant correlation $(r=0.508$, $P<0.002)$ was also found between the habits of running and the differences in the degrees of the calcaneal vertical angle on the right foot, meaning that the amount of eversion was higher in the low arches and increased more after the running session.

\section{DISCUSSION}

The aim of the present study was to investigate the effects of fatigue and its contribution to new unrevealed information about abnormal foot structures, abnormal biomechanics, and factors that predispose one to injuries. The different effects of fatigue for the PP and the PC groups, found in the present study, could indicate that the differences between the PC and PP dynamic behavior do in fact exist, but first in a fatigue state. Both the PC and the PP groups showed an increased eversion after the 45-min running session, which supports our hypothesis. The hypothesis of fatigue affection was made upon presumptions that prolonged running would cause muscle fatigue and decrease the efficiency of the antipronatory muscles. The results are in agreement with earlier studies testing the effects of running induced fatigue on rearfoot motion (Clansey et al., 2012; Derrick et al., 2002; Dierks et al., 2010). Clansey et al. (2012) showed that there was an increase in the peak rearfoot eversion after two periods of 20-min running under the lactate threshold. Derrick et al. (2002) also found a significant increase in the maximal rearfoot eversion, as much as $20 \%$ after a 3,200-m pace. As in the present study, Dierks et al. (2010) discuss the importance of simulating the individual's typi- cal running session. Therefore, they investigated subjects running on a treadmill at a self-selected speed until they reached their typical training level of exhaustion. The same results were found in the high intensity studies (Clansey et al., 2012; Derrick et al., 2002; Dierks et al., 2010). However, the results in the present study are in contrast to that of Abt et al. (2011) and Hamill et al. (1988) where they found no effects from fatigue on the rearfoot motion during midstance, using high intensity running.

In order to collect potential information about the complexities of fatigue, several individual parameters were tested for correlations with the STJ motion. The general exhaustion, as rated on the RPE Borg scale (Borg, 1998), did not show any correlation to the altered kinematics, suggesting that the individual differences of the fatigue effect might be found in the local foot. Ferber and Pohl (2011) have investigated the importance of fatigue of $\mathrm{Mm}$. Tibialis posterior, by using isolated muscle work but no significant difference was found on the running step (Ferber and Pohl 2011). The passive plantar structures have also been described as being of importance for the MLA (Jastifer and Gustafson, 2014). A creep might contribute to an increased eversion of the STJ. More studies investigating the active and passive structures' reaction on fatigue might contribute to further understanding of the complexities of the foot in relation to fatigue.

The second hypothesis in the present study was that PP would be more affected by fatigue, showing an increased amount of post run eversion compared to PC. Since there were no significant differences between the PP and PC, after 5 min of running, it would indicate that those with PP are more affected by fatigue. The rea- 
son for a side discrepancy between the right and the left foot is unknown. Due to the lack of similar studies, the hypothesis that PP would be more affected by fatigue was made through presumptions. The MLA-angle has been associated with a larger deformation in the midstance during running (McPoil and Cornwall, 2005), which would indicate a greater MLA deformation in the PP than the PC. The increased MLA-deformation might increase the creep in the passive structures and thereby increase fatigue in the dynamic stabilizers of the arch, responsible for anteversion of the STJ. One study measured the static foot postures during bilateral standing before and after a half marathon run (Cowley and Marsden, 2013). Subjects with a higher prerun n-height showed a significantly greater drop postrun. However, Cowley and Marsden (2013) discussed the nonperformed normalization of the $\mathrm{n}$-height since larger feet show higher values and are more sensitive to changes.

The present study showed no significant differences between non fatigue PC and PP. To the best of our knowledge, no similar studies have been done investigating the relationship between $2 \mathrm{D}$ freeze frame images of the CV-angle and the MLA-angle. Two previous studies used the same static MLA-angle and found no prediction of a three-dimensional (3D) rearfoot eversion either during walking or running (Hunt et al., 2000). Earlier studies using different methods have shown some correlations during running (Lee and Hertel, 2012), while others have shown a lack of correlations during walking (Hunt et al., 2000), and running (Nigg et al., 1993). However, these studies report no information about the foot structure being related to the abnormal biomechanics. Studies investigating the differences in defined groups of PP and PC found significantly greater and delayed rearfoot motion in the PP during running, compared to the PC (Barnes et al., 2011). According to a systematic review by Buldt et al. (2013) there was some evidence for increased rearfoot eversion during gait, in a defined group of PP feet, but this was limited by small effect sizes. Studies investigating the effect of defined groups of $\mathrm{PP}$ and PC on the subtalar motion data might be a lot more useful in the clinics, giving clinicians a quick tool to separate the abnormal foot structures from the normal ones and predict abnormal dynamic behavior in order to find a suitable treatment. However, a limitation of the present study is the exclusion of the PP. There seems to be a lack of investigations including all three-foot structures in earlier running research. Since both high and low arches have been shown to have an increased risk of injury compared to the normal arches (Kaufman et al., 1999), a comparison of the PR would be of great interest. The reasons for the higher risks in the
PC and PP have been found to be due to the abnormal pronation for the PP and the decreased shock attenuation ability in the PC (Williams et al., 2014).

The foot mobility is a somewhat new and interesting characterization of the foot suggested to explain the dynamic behavior in the independency of the foot structure (Williams et al., 2014). Mobile HA runners have shown different compensation patterns closer to the LA, with increased eversion and decreased impact load (Williams et al., 2014).

Repetitive impact forces are encountered during running and can lead to overuse injuries. The STJ motion is important for shock attenuation and the increased eversion in both the PC and the PP seen in the fatigue state might have an impact on predisposing injuries. However, Abt et al. (2011) found no significant changes for either rearfoot or shock attenuation in women distance runners. Clansey et al. (2012) showed an increase in both the eversion and the impact load in well trained and competitive distance runners. Derrick et al. (2002) found an increased eversion and decreased impact load, hence, increased shock attenuation in recreation runners during a prolonged run. Thus, there are contrasting results as to whether or not an increased eversion is an optimal STJ motion to reduce the impact during a prolonged run. Higher shock attenuation is associated with higher oxygen cost (Derrick et al., 2002). In the present study, the individual's own experience of running habits was the only parameter impacted by fatigue, showing that decreased running habits resulted in a significantly greater amount of eversion and also significantly increased eversion postrun compared to prerun, on the right foot. Further research is needed to find out more about the complexities of running induced fatigue and its links to injuries.

The MLA-angle has been highlighted, compared to anthropometric measurements, since it composes both the arch-height and the length (Williams and McClay, 2000). The skeleton reference points of the MLA-angle have also been stated as being more valid compared to the measurement along the foot's soft tissue (McPoil and Cornwall, 2005; Williams and McClay, 2000). During the measurement of the MLA-angle, the subject stood on one leg. Less bodyweight, such as $10 \%$, has shown higher reliability, suggesting more body weight complicates the palpation (Williams and McClay, 2000). Although the reference points are relatively easy to identify and well used clinical palpation points, it is, however, surprising how nonspecifically the reference points are described in previous studies (Nilsson et al., 2012).

In the present study, further potential sources of error have been taken into consideration, for example, the use of a fine ballpoint 
pen since thickness of the pen has been shown to be significant with the degree of errors. Furthermore, the center line was marked only once in each subject. The examiner used a pen-marked center line of the calcaneus, while numerous studies have used a hypothetical midline during the angle measurements (Elveru et al., 1988; Stacpoole-Shea et al., 1998).

The digital goniometry used for both the MLA-angle and the $\mathrm{CV}$-angle has practical advantages, as the reference lines could be added one at a time and be rectified. Borel et al. (2011) used Dartfish for measurements of the knee flexion during the midstance gait and showed improved reliability, compared to visually observing the subjects in Windows media player.

A potential limitation of the study is the validation between running with shoes on and barefoot running. The research presents an ongoing debate about this, however, concerning the rearfoot a systematic review by Hall et al. (2013) shows that there is very limited evidence that the amount of eversion or maximal eversion angle is reduced or occurs earlier during the barefoot running compared to running with shoes. Since having a barefoot subject also simplifies the use of external markings, it seems sensible to use subjects without shoes. As in similar earlier studies, the subjects used their own running shoes during the 45-min run. They were informed not to use a minimalistic shoe, thus, various motion control, and cushioning shoes from different brands were used. However, even if the possibility of shoe affection between individuals exists, running with shoes on might also have more accurately reflected a real-life situation for each runner.

Another potential limitation of the study is assuming that the values obtained from the $\mathrm{CV}$-angle represent the movements at the STJ, eversion/inversion. Even if Cornwall and McPoil (1995) has demonstrated that the differences between the $2 \mathrm{D}$ and $3 \mathrm{D}$ analysis of rearfoot motion are minimal, if the stance-phase does not extend over the first $60 \%$, it will always be a risk to use simple measurement tools to explain the complex biomechanical systems. However, other equally important aspects that symbolize good clinical evaluation methods besides validity and reliability are clinical usefulness. The 2D analysis used in the present study is time effective and requires a relatively simple computer, Dartfish Software, and a video camera, in contrast to the high cost of the equipment in 3D analysis. The 2D analysis with digital angle measurements has also shown acceptable reliability both in the present and earlier studies; moreover, it is better than both visual assessment and standard goniometry frequently used in daily clinics (McPoil and Cornwall, 2005). More studies of reliability and validity against $3 \mathrm{D}$ analysis are needed to establish the use of $2 \mathrm{D}$ analysis and digital angle measurement programs in the clinic. In order to establish better quality evidence, it is also desirable to develop a greater consensus for the kinematic foot measurement in future research.

In summary, our results demonstrate that static measurements of the MLA-angle would not be of assistance in predicting the biomechanical patterns of the subtalar joint (CV-angle) during running. However the PP and PC displayed an increased eversion motion in the STJ after 45 min of running, compared to a few minutes of running, which questions the validity of using a short running analysis in biomechanical research. More studies investigating the running induced fatigue are needed to clarify the relationship between foot structures, biomechanical patterns, and factors that predispose one to injuries.

\section{CONFLICT OF INTEREST}

No potential conflict of interest relevant to this article was reported.

\section{REFERENCES}

Abt JP, Sell TC, Chu Y, Lovalekar M, Burdett RG, Lephart SM. Running kinematics and shock absorption do not change after brief exhaustive running. J Strength Cond Res 2011;25:1479-1485.

Barnes A, Wheat J, Milner CE. Fore- and rearfoot kinematics in high- and low-arched individuals during running. Foot Ankle Int 2011;32:710716.

Borel S, Schneider P, Newman CJ. Video analysis software increases the interrater reliability of video gait assessments in children with cerebral palsy. Gait Posture 2011;33:727-729.

Borg G. Borg's perceived exertion and pain scales. Champaign (IL): Human Kinetics;1998.

Buldt AK, Murley GS, Butterworth P, Levinger P, Menz HB, Landorf KB. The relationship between foot posture and lower limb kinematics during walking: a systematic review. Gait Posture 2013;38:363-372.

Clansey AC, Hanlon M, Wallace ES, Lake MJ. Effects of fatigue on running mechanics associated with tibial stress fracture risk. Med Sci Sports Exerc 2012;44:1917-1923.

Cornwall MW, McPoil TG. Comparison of 2-dimensional and 3-dimensional rearfoot motion during walking. Clin Biomech (Bristol, Avon) 1995;10:36-40.

Cowley E, Marsden J. The effects of prolonged running on foot posture: a repeated measures study of half marathon runners using the foot posture index and navicular height. J Foot Ankle Res 2013;6:20. 
Dahle LK, Mueller MJ, Delitto A, Diamond JE. Visual assessment of foot type and relationship of foot type to lower extremity injury. J Orthop Sports Phys Ther 1991;14:70-74.

Derrick TR, Dereu D, McLean SP. Impacts and kinematic adjustments during an exhaustive run. Med Sci Sports Exerc 2002;34:998-1002.

Dierks TA, Davis IS, Hamill J. The effects of running in an exerted state on lower extremity kinematics and joint timing. J Biomech 2010;43: 2993-2998.

Dierks TA, Manal KT, Hamill J, Davis I. Lower extremity kinematics in runners with patellofemoral pain during a prolonged run. Med Sci Sports Exerc 2011;43:693-700.

Elveru RA, Rothstein JM, Lamb RL, Riddle DL. Methods for taking subtalar joint measurements. A clinical report. Phys Ther 1988;68:678-682.

Ferber R, Pohl MB. Changes in joint coupling and variability during walking following tibialis posterior muscle fatigue. J Foot Ankle Res 2011;4:6.

Hall JP, Barton C, Jones PR, Morrissey D. The biomechanical differences between barefoot and shod distance running: a systematic review and preliminary meta-analysis. Sports Med 2013;43:1335-1353.

Hamill J, Freedson PS, Boda W, Reichsman F. Effects of shoe type on cardiorespiratory responses and rearfoot motion during treadmill running. Med Sci Sports Exerc 1988;20:515-521.

Hunt AE, Fahey AJ, Smith RM. Static measures of calcaneal deviation and arch angle as predictors of rearfoot motion during walking. Aust J Physiother 2000;46:9-16.

Jastifer JR, Gustafson PA. The subtalar joint: biomechanics and functional representations in the literature. Foot (Edinb) 2014;24:203-209.

Johanson MA, Donatelli R, Wooden MJ, Andrew PD, Cummings GS. Effects of three different posting methods on controlling abnormal subtalar pronation. Phys Ther 1994;74:149-158.
Kannus VP. Evaluation of abnormal biomechanics of the foot and ankle in athletes. Br J Sports Med 1992;26:83-89.

Kaufman KR, Brodine SK, Shaffer RA, Johnson CW, Cullison TR. The effect of foot structure and range of motion on musculoskeletal overuse injuries. Am J Sports Med 1999;27:585-593.

Lee SY, Hertel J. Arch height and maximum rearfoot eversion during jogging in 2 static neutral positions. J Athl Train 2012;47:83-90.

McPoil TG, Cornwall MW. Use of the longitudinal arch angle to predict dynamic foot posture in walking. J Am Podiatr Med Assoc 2005;95: 114-120.

Nigg BM, Cole GK, Nachbauer W. Effects of arch height of the foot on angular motion of the lower extremities in running. J Biomech 1993; 26:909-916.

Nilsson MK, Friis R, Michaelsen MS, Jakobsen PA, Nielsen RO. Classification of the height and flexibility of the medial longitudinal arch of the foot. J Foot Ankle Res 2012;5:3.

Razeghi M, Batt ME. Foot type classification: a critical review of current methods. Gait Posture 2002;15:282-291.

Stacpoole-Shea S, Shea G, Otago L, Payne W. Instrumentation considerations of a clinical and a computerized technique for the measurement of foot angles. J Foot Ankle Surg 1998;37:410-415.

Subotnick SI. The subtalar joint lateral extra-articular arthroereisis: a preliminary report. J Am Podiatry Assoc 1974;64:701-711.

Williams DS, McClay IS. Measurements used to characterize the foot and the medial longitudinal arch: reliability and validity. Phys Ther 2000; 80:864-871.

Williams DS 3rd, Tierney RN, Butler RJ. Increased medial longitudinal arch mobility, lower extremity kinematics, and ground reaction forces in high-arched runners. J Athl Train 2014;49:290-296. 